Annals of Pure and Applied Mathematics

Vol. 16, No. 1, 2018, 7-19

ISSN: 2279-087X (P), 2279-0888(online)

Published on 1 January 2018

www.researchmathsci.org

DOI: http://dx.doi.org/10.22457/apam.v16n1a2

Annals of

Pure and Applied

Mathematics

\title{
Asymptotics of Eigenvalues for Sturm-Liouville Problem with Eigenvalue in the Boundary Condition for Differentiable Potential
}

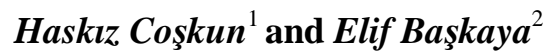 \\ ${ }^{1}$ Department of Mathematics, Karadeniz Technical University \\ TR - 61080 Trabzon.E-mail: haskiz@ ktu.edu.tr \\ ${ }^{2}$ Department of Mathematics, Karadeniz Technical University \\ TR - 61080 Trabzon.E-mail: elifbekar@ktu.edu.tr \\ Received 3November 17; accepted 2 December 2017
}

Abstract. In this paper, we obtain asymptotic estimates of eigenvalues for regular SturmLiouville problems having the eigenvalue parameter in the boundary condition with the potential that is continuous, also its differentiation exists and is integrable.

Keywords: Sturm-liouville problems; differentiable potential; eigenvalues; asymptotics.

AMS Mathematics Subject Classification (2010): 34B08,34B24, 34E05

\section{Introduction}

In this paper, we consider the boundary value problem

$$
\begin{array}{r}
y^{\prime \prime}(t)+\{\lambda-q(t)\} y(t)=0, \quad t \in[a, b], \\
a_{1} y(a)-a_{2} y^{\prime}(a)=\lambda\left[a_{1}^{\prime} y(a)-a_{2}^{\prime} y^{\prime}(a)\right], \\
y(b) \cos \beta+y^{\prime}(b) \sin \beta=0, \quad \beta \in[0, \pi),
\end{array}
$$

where $\lambda$ is a real parameter; $q(t)$ is a real-valued function; $a_{1}, a_{2}, a_{1}^{\prime}, a_{2}^{\prime} \in \mathbb{R}$. Also we assume that $\mathrm{q}(\mathrm{t})$ is continuous, its differentiation exists and is integrable. This problem differs from the usual regular Sturm-Liouville problem in the sense that eigenvalue parameter $\lambda$ is contained in the boundary condition at a . Problems of this type arise from the method of separation of variables applied to mathematical models for certain physical problems including heat conduction and wave propagation, etc. [8]. It is shown by Walter [15] that this problem is self-adjoint problem if the relation $a_{1}^{\prime} a_{2}-a_{1} a_{2}^{\prime}>0$. The purpose of this paper is to obtain asymptotic approximations for the eigenvalues of (1)(3).

Approximations of this type have been derived before. We mention in particular [7], [8] and [2]. Fulton's approach in [7] is based on an iteration of the usual Volterra integral equation, producing an asymptotic expansion of the solution in higher powers of $1 / \lambda^{1 / 2}$ as $\lambda \rightarrow \infty$ and in [8] is based on the analysis of [14] for regular Sturm-Liouville problems on a finite closed interval and involves some operator-theoretical results of [15]. The 


\section{Haskız Coşkun and Elif Başkaya}

approach used in [2] is based on an iterative procedure solving the associated Riccati equation and producing an asymptotic expansion of the solution in the higher powers of $1 / \lambda^{1 / 2}$ as $\lambda \rightarrow \infty$ for smooth $\mathrm{q}(\mathrm{t})$. There is also a vast amount of literature dealing with asymptotic estimates of eigenvalues for standard Sturm-Liouville problems with regular endpoints $[3,4,5,6,9,10,11,13,14]$. Here we follow the similar approach in $[4,10,12]$. We assume without loss of generality, that $q(t)$ has mean value zero. That is $\int_{a}^{b} q(t) d t=0$.

\section{Results}

In this study, we gain the following results:

Theorem 2.1.The eigenvalues $\lambda_{\mathrm{n}}$ of (1)-(3) satisfy as $\lambda \rightarrow \infty$,

i) if $a_{2}^{\prime} \neq 0$ and $\beta \neq 0$,

$$
\begin{aligned}
\lambda_{n}^{1 / 2} & =\frac{(n+1) \pi}{b-a}+\frac{1}{(n+1) \pi}\left\{\cot \beta+\frac{a_{1}^{\prime}}{a_{2}^{\prime}}-\frac{b-a}{4(n+1) \pi} \int_{a}^{b}\left[\sin \frac{2(n+1) \pi(x-a)}{b-a}\right] q^{\prime}(x) d x\right. \\
& +\frac{a_{1}^{\prime}}{2 a_{2}^{\prime}} \frac{(b-a)^{2}}{(n+1)^{2} \pi^{2}}\left[q(a)-q(b)-\frac{2\left(3 a_{1}^{\prime} a_{2}^{\prime} a_{2}+3 a_{1}\left[a_{2}^{\prime}\right]^{2}+\left[a_{1}^{\prime}\right]^{3}\right)}{3 a_{1}^{\prime}\left[a_{2}^{\prime}\right]^{2}}-\frac{2 a_{2}^{\prime}}{3 a_{1}^{\prime}} \cot ^{3} \beta\right] \\
& \left.+\frac{a_{1}^{\prime}}{2 a_{2}^{\prime}} \frac{(b-a)^{2}}{(n+1)^{2} \pi^{2}} \int_{a}^{b}\left[\cos \frac{2(n+1) \pi(x-a)}{b-a}\right] q^{\prime}(x) d x\right\}+O\left(n^{-4} \eta(n)\right)+O\left(n^{-3} \eta^{2}(n)\right), \\
\text { ii) } & \\
\lambda_{n}^{1 / 2}= & \frac{(2 n+3) \pi}{2(b-a)}+\frac{1}{(2 n+3) \pi}\left\{\frac{2 a_{1}^{\prime}}{a_{2}^{\prime}}-\frac{b-a}{(2 n+3) \pi} \int_{a}^{b}\left[\sin \frac{(2 n+3) \pi(x-a)}{b-a}\right] q^{\prime}(x) d x\right. \\
& +\frac{4 a_{1}^{\prime}}{a_{2}^{\prime}} \frac{(b-a)^{2}}{(2 n+3)^{2} \pi^{2}}\left[q(a)+q(b)+\frac{2\left(3 a_{1}^{\prime} a_{2}^{\prime} a_{2}-3 a_{1}\left[a_{2}^{\prime}\right]^{2}-\left[a_{1}^{\prime}\right]^{3}\right)}{3 a_{1}^{\prime}\left[a_{2}^{\prime}\right]^{2}}\right] \\
& \left.+\frac{4 a_{1}^{\prime}}{a_{2}^{\prime}} \frac{(b-a)^{2}}{(2 n+3)^{2} \pi^{2}} \int_{a}^{b}\left[\cos \frac{(2 n+3) \pi(x-a)}{b-a}\right] q^{\prime}(x) d x\right\}+O\left(n^{-4} \eta(n)\right)+O\left(n^{-3} \eta^{2}(n)\right) .
\end{aligned}
$$

Theorem 2.2.The eigenvalues $\lambda_{\mathrm{n}}$ of (1)-(3) satisfy as $\lambda \rightarrow \infty$,

i) if $a_{2}^{\prime}=0$ and $\beta \neq 0$, 
Asymptotics of Eigenvalues for Sturm-Liouville Problem with Eigenvalue in the Boundary Condition for Differentiable Potential

$$
\begin{aligned}
& \lambda_{\mathrm{n}}^{1 / 2}=\frac{(2 \mathrm{n}+3) \pi}{2(\mathrm{~b}-\mathrm{a})}+\frac{1}{(2 \mathrm{n}+3) \pi}\left\{2 \cot \beta+\frac{2 \mathrm{a}_{2}}{\mathrm{a}_{1}^{\prime}}+\frac{\mathrm{b}-\mathrm{a}}{(2 \mathrm{n}+3) \pi} \int_{\mathrm{a}}^{\mathrm{b}}\left[\sin \frac{(2 \mathrm{n}+3) \pi(\mathrm{x}-\mathrm{a})}{\mathrm{b}-\mathrm{a}}\right] \mathrm{q}^{\prime}(\mathrm{x}) \mathrm{dx}\right. \\
& -\frac{4 a_{2}}{a_{1}^{\prime}} \frac{(b-a)^{2}}{(2 n+3)^{2} \pi^{2}}\left[q(a)+q(b)+\frac{2\left(1-\frac{3 a_{1}^{\prime} a_{1}}{a_{2}^{2}}\right)}{3\left[\frac{a_{1}^{\prime}}{a_{2}}\right]^{2}}-\frac{2}{3} \cot ^{3} \beta\right] \\
& \left.-\frac{4 a_{2}}{a_{1}^{\prime}} \frac{(b-a)^{2}}{(2 n+3)^{2} \pi^{2}} \int_{a}^{b}\left[\cos \frac{(2 n+3) \pi(x-a)}{b-a}\right] q^{\prime}(x) d x\right\}+O\left(n^{-4} \eta(n)\right)+O\left(n^{-3} \eta^{2}(n)\right),
\end{aligned}
$$

ii) if $a_{2}^{\prime}=0$ and $\beta=0$,

$$
\begin{aligned}
& \lambda_{\mathrm{n}}^{1 / 2}=\frac{(\mathrm{n}+2) \pi}{\mathrm{b}-\mathrm{a}}+\frac{1}{(\mathrm{n}+2) \pi}\left\{\frac{\mathrm{a}_{2}}{\mathrm{a}_{1}^{\prime}}+\frac{\mathrm{b}-\mathrm{a}}{4(\mathrm{n}+2) \pi} \int_{\mathrm{a}}^{\mathrm{b}}\left[\sin \frac{2(\mathrm{n}+2) \pi(\mathrm{x}-\mathrm{a})}{\mathrm{b}-\mathrm{a}}\right] \mathrm{q}^{\prime}(\mathrm{x}) \mathrm{dx}\right. \\
& -\frac{a_{2}}{2 a_{1}^{\prime}} \frac{(b-a)^{2}}{(n+2)^{2} \pi^{2}}\left[q(a)-q(b)+\frac{2\left(1-\frac{3 a_{1}^{\prime} a_{1}}{a_{2}^{2}}\right)}{3\left[\frac{a_{1}^{\prime}}{a_{2}}\right]^{2}}\right] \\
& \left.-\frac{a_{2}}{2 a_{1}^{\prime}} \frac{(b-a)^{2}}{(n+2)^{2} \pi^{2}} \int_{a}^{b}\left[\cos \frac{2(n+2) \pi(x-a)}{b-a}\right] q^{\prime}(x) d x\right\}+O\left(n^{-4} \eta(n)\right)+O\left(n^{-3} \eta^{2}(n)\right) \text {. }
\end{aligned}
$$

\section{The method}

We associate with (1) the Riccati equation

$$
\mathrm{v}^{\prime}(\mathrm{t}, \lambda)=-\lambda+\mathrm{q}-\mathrm{v}^{2} .
$$

We define

$$
\begin{aligned}
& \mathrm{S}(\mathrm{t}, \lambda)=\operatorname{Re}[\mathrm{v}(\mathrm{t}, \lambda)], \\
& \mathrm{T}(\mathrm{t}, \lambda)=\operatorname{Im}[\mathrm{v}(\mathrm{t}, \lambda)] .
\end{aligned}
$$

It is shown in [3] that any real-valued solution of (1) is in the form

$$
\mathrm{y}(\mathrm{t}, \lambda)=\mathrm{R}(\mathrm{t}, \lambda) \cos \theta(\mathrm{t}, \lambda)
$$

with

$$
\begin{aligned}
& S(t, \lambda)=\frac{R^{\prime}(t, \lambda)}{R(t, \lambda)}, \\
& T(t, \lambda)=\theta^{\prime}(t, \lambda) .
\end{aligned}
$$

Our approach to calculating $\lambda_{\mathrm{n}}$ is to approximate those $\lambda$ which are such that 


\section{Haskız Coşkun and Elif Başkaya}

$$
\theta(b, \lambda)-\theta(a, \lambda)=\int_{a}^{b} T(x, \lambda) d x
$$

by the last equation.

We suppose that there exist functions $A(t)$ and $\eta(\lambda)$ so that

where

$$
\left|\int_{t}^{b} e^{2 i \lambda^{1 / 2} x} q^{\prime}(x) d x\right| \leq A(t) \eta(\lambda), \quad t \in[a, b]
$$

i) $A(t):=\int_{t}^{b}\left|q^{\prime}(x)\right| d x$ is a decreasing function of $t$,

ii) $\mathrm{A}(.) \in \mathrm{L}[\mathrm{a}, \mathrm{b}]$,

iii) $\eta(\lambda) \rightarrow 0$ as $\lambda^{1 / 2} \rightarrow \infty$.

For $\mathrm{q}^{\prime} \in \mathrm{L}[\mathrm{a}, \mathrm{b}]$ the existence of the $\mathrm{A}$ and $\eta$ functions may be established for $\lambda$ positive as follows. We note that, avoiding the trivial case $\int_{t}^{b}\left|q^{\prime}(x)\right| d x=0$.

$$
\begin{aligned}
\left|\int_{t}^{b} e^{2 i \lambda^{\prime / 2}} q^{\prime}(x) d x\right| \leq \int_{t}^{b}\left|q^{\prime}(x)\right| d x<\infty \text { so, if we define } \\
F(t, \lambda):= \begin{cases}\left|\int_{t}^{b} e^{2 i \lambda^{1 / 2} x} q^{\prime}(x) d x\right| / \int_{t}^{b}\left|q^{\prime}(x)\right| d x, & \text { if } \int_{t}^{b}\left|q^{\prime}(x)\right| d x \neq 0, \\
0 & , \text { if } \int_{t}^{b}\left|q^{\prime}(x)\right| d x=0,\end{cases}
\end{aligned}
$$

then $0 \leq F(t, \lambda) \leq 1$ and we set $\eta(\lambda):=\sup _{a \leq t \leq b} F(t, \lambda)$. $\eta(\lambda)$ is well defined by (10) and $\lambda^{-1 / 2} \eta(\lambda) \rightarrow 0$ as $\lambda \rightarrow \infty[12]$.

Our method of approximating a solution of Riccati equation $v^{\prime}(t, \lambda)=-\lambda+q-v^{2}$ on [a,b] is similar to [12], so we set

$$
v(t, \lambda):=i \lambda^{1 / 2}+\sum_{n=1}^{\infty} v_{n}(t, \lambda) .
$$

When we put this serieinto the Riccati equation and solve differential equations, we hold 
Asymptotics of Eigenvalues for Sturm-Liouville Problem with Eigenvalue in the Boundary Condition for Differentiable Potential

$$
\begin{aligned}
& \mathrm{v}_{1}(\mathrm{t}, \lambda)=-\mathrm{e}^{-2 i \lambda^{1 / 2} \mathrm{t}} \int_{t}^{\mathrm{b}} \mathrm{e}^{2 i \lambda^{1 / 2} \mathrm{x}} \mathrm{q}(\mathrm{x}) \mathrm{dx}, \\
& \mathrm{v}_{2}(\mathrm{t}, \lambda)=\mathrm{e}^{-2 \mathrm{i} \lambda^{1 / 2}} \int_{\mathrm{t}}^{\mathrm{b}} \mathrm{e}^{2 \mathrm{i} \lambda^{1 / 2} \mathrm{x}} \mathrm{v}_{1}^{2}(\mathrm{x}, \lambda) \mathrm{dx}, \\
& \mathrm{v}_{\mathrm{n}}(\mathrm{t}, \lambda)=\mathrm{e}^{-2 \mathrm{i} \lambda^{1 / 2}} \int_{\mathrm{t}}^{\mathrm{b}} \mathrm{e}^{2 \mathrm{i} \lambda^{1 / 2} \mathrm{x}}\left[\mathrm{v}_{\mathrm{n}-1}^{2}(\mathrm{x}, \lambda)+2 \mathrm{v}_{\mathrm{n}-1}(\mathrm{x}, \lambda) \sum_{\mathrm{m}=1}^{\mathrm{n}-2} \mathrm{v}_{\mathrm{m}}(\mathrm{x}, \lambda)\right] \mathrm{dx}, \mathrm{n} \geq 3 .
\end{aligned}
$$

Also we found $\theta(b, \lambda)-\theta(a, \lambda)=\int_{a}^{b} T(x, \lambda) d x$, so with (5) and (11), we have

$$
\begin{aligned}
\theta(b, \lambda)-\theta(a, \lambda)=\int_{a}^{b}\left[\lambda^{1 / 2}+\operatorname{Im} \sum_{n=1}^{\infty} v_{n}(x, \lambda)\right] d x \text {, then } \\
\\
\quad \theta(b, \lambda)-\theta(a, \lambda)=\lambda^{1 / 2}(b-a)+\sum_{n=1}^{\infty} \operatorname{Im} \int_{a}^{b} v_{n}(x, \lambda) d x .
\end{aligned}
$$

Theorem 3.1. [1] If $v(t, \lambda)$ as in (11), as $\lambda \rightarrow \infty$

$$
\mathrm{v}(\mathrm{t}, \lambda)=\mathrm{i} \lambda^{1 / 2}+\mathrm{v}_{1}(\mathrm{t}, \lambda)+\mathrm{O}\left(\lambda^{-1} \eta^{2}(\lambda)\right)
$$

where

$$
\begin{aligned}
\mathrm{v}_{1}(\mathrm{t}, \lambda) & =-\frac{1}{2} \lambda^{-1 / 2} \mathrm{q}(\mathrm{b}) \sin 2 \lambda^{1 / 2}(\mathrm{~b}-\mathrm{t})+\frac{1}{2} \lambda^{-1 / 2} \int_{\mathrm{t}}^{\mathrm{b}}\left[\sin 2 \lambda^{1 / 2}(\mathrm{x}-\mathrm{t})\right] \mathrm{g}^{\prime}(\mathrm{x}) \mathrm{dx} \\
& +\mathrm{i}\left\{\frac{1}{2} \lambda^{-1 / 2} \mathrm{q}(\mathrm{b}) \cos 2 \lambda^{1 / 2}(\mathrm{~b}-\mathrm{t})-\frac{1}{2} \lambda^{-1 / 2} \mathrm{q}(\mathrm{t})-\frac{1}{2} \lambda^{-1 / 2} \int_{\mathrm{t}}^{\mathrm{b}}\left[\cos 2 \lambda^{1 / 2}(\mathrm{x}-\mathrm{t})\right] \mathrm{q}^{\prime}(\mathrm{x}) \mathrm{dx}\right\}
\end{aligned}
$$

and $\eta(\lambda)$ is defined (10).

After some calculations by using the last theorem, with (4) we gain

$$
\begin{aligned}
S(t, \lambda) & =-\frac{1}{2} \lambda^{-1 / 2} q(b) \sin 2 \lambda^{1 / 2}(b-t)+\frac{1}{2} \lambda^{-1 / 2}\left(\cos 2 \lambda^{1 / 2} t\right) \int_{t}^{b}\left[\sin 2 \lambda^{1 / 2} x\right] q^{\prime}(x) d x \\
& -\frac{1}{2} \lambda^{-1 / 2}\left(\sin 2 \lambda^{1 / 2} t\right) \int_{t}^{b}\left[\cos 2 \lambda^{1 / 2} x\right] g^{\prime}(x) d x+O\left(\lambda^{-1} \eta^{2}(\lambda)\right) .
\end{aligned}
$$

Let define thefollowingnotations:

$$
\begin{aligned}
& \sin \xi_{\mathrm{t}}:=\int_{\mathrm{t}}^{\mathrm{b}}\left[\cos 2 \lambda^{1 / 2} \mathrm{x}\right] \mathrm{q}^{\prime}(\mathrm{x}) \mathrm{dx}, \\
& \cos \xi_{\mathrm{t}}:=\int_{\mathrm{t}}^{\mathrm{b}}\left[\sin 2 \lambda^{1 / 2} \mathrm{x}\right] \mathrm{q}^{\prime}(\mathrm{x}) \mathrm{dx},
\end{aligned}
$$

thuswe can write $S(t, \lambda)$ as

$$
S(t, \lambda)=-\frac{1}{2} \lambda^{-1 / 2} q(b) \sin 2 \lambda^{1 / 2}(b-t)+\frac{1}{2} \lambda^{-1 / 2}\left(\cos 2 \lambda^{1 / 2} t+\xi_{t}\right)+O\left(\lambda^{-1} \eta^{2}(\lambda)\right) .
$$




\section{Haskız Coşkun and Elif Başkaya}

Similarly, with (5) we find $\mathrm{T}(\mathrm{t}, \lambda)$ as

$$
\begin{aligned}
\mathrm{T}(\mathrm{t}, \lambda) & =\lambda^{1 / 2}+\frac{1}{2} \lambda^{-1 / 2} \mathrm{q}(\mathrm{b}) \cos 2 \lambda^{1 / 2}(\mathrm{~b}-\mathrm{t})-\frac{1}{2} \lambda^{-1 / 2} \mathrm{q}(\mathrm{t})-\frac{1}{2} \lambda^{-1 / 2}\left(\sin 2 \lambda^{1 / 2} \mathrm{t}+\xi_{\mathrm{t}}\right) \\
& +\mathrm{O}\left(\lambda^{-1} \eta^{2}(\lambda)\right) .
\end{aligned}
$$

Also, by using integration by part to (12), we determine

$$
\int_{a}^{b} v_{1}(x, \lambda) d x=\frac{i}{2 \lambda^{1 / 2}} \int_{t}^{b} e^{2 i \lambda^{1 / 2}(x-a)} q(x) d x
$$

and again with integration by part

$$
\begin{aligned}
\int_{a}^{b} v_{1}(x, \lambda) d x & =\frac{i}{2} \lambda^{-1 / 2} e^{-2 i \lambda^{1 / 2} a}\left[\left.\frac{q(x) e^{2 i \lambda^{1 / 2} x}}{2 i \lambda^{1 / 2}}\right|_{x=a} ^{b}-\frac{1}{2 i \lambda^{1 / 2}} \int_{a}^{b} e^{2 i \lambda^{1 / 2}} q^{\prime}(x) d x\right] \\
& =\frac{1}{4} \lambda^{-1} e^{2 i \lambda^{1 / 2}(b-a)} q(b)-\frac{1}{4} \lambda^{-1} q(a)-\frac{1}{4} \lambda^{-1} e^{-2 i \lambda^{1 / 2}} \int_{a}^{b} e^{2 i \lambda^{1 / 2}} q^{\prime}(x) d x \\
& =\frac{1}{4} \lambda^{-1} q(b)\left[\cos 2 \lambda^{1 / 2}(b-a)+i \sin 2 \lambda^{1 / 2}(b-a)\right]-\frac{1}{4} \lambda^{-1} q(a) \\
& -\frac{1}{4} \lambda^{-1} \int_{a}^{b}\left[\cos 2 \lambda^{1 / 2}(x-a)+i \sin 2 \lambda^{1 / 2}(x-a)\right] q^{\prime}(x) d x,
\end{aligned}
$$

so

$$
\operatorname{Im} \int_{a}^{b} v_{1}(x, \lambda) d x=\frac{1}{4} \lambda^{-1} q(b) \sin 2 \lambda^{1 / 2}(b-a)-\frac{1}{4} \lambda^{-1} \cos \left(2 \lambda^{1 / 2} a+\xi_{a}\right) .
$$

We also have from equation (12),

$$
\int_{a}^{b} v_{2}(x, \lambda) d x=\frac{i}{2 \lambda^{1 / 2}} \int_{a}^{b}\left[1-e^{2 i \lambda^{1 / 2}(x-a)}\right] v_{1}^{2}(x, \lambda) d x
$$

and for $\mathrm{n} \geq 3$

$$
\int_{a}^{b} v_{n}(x, \lambda) d x=\frac{i}{2 \lambda^{1 / 2}} \int_{a}^{b}\left[1-e^{2 i \lambda^{1 / 2}(x-a)}\right]\left[v_{n-1}^{2}(x, \lambda)+2 v_{n-1}(x, \lambda) \sum_{m=1}^{n-2} v_{m}(x, \lambda)\right] d x .
$$

Thus, with the last equations

$$
\begin{aligned}
\int_{a}^{b} \sum_{n=1}^{\infty} \operatorname{Im}\left\{v_{n}(x, \lambda)\right\} d x & =\sum_{n=1}^{\infty} \operatorname{Im}\left\{\int_{a}^{b} v_{n}(x, \lambda) d x\right\} \\
& =\frac{1}{4} \lambda^{-1} q(b) \sin 2 \lambda^{1 / 2}(b-a)-\frac{1}{4} \lambda^{-1} \cos \left(2 \lambda^{1 / 2} a+\xi_{a}\right) \\
& +O\left(\lambda^{-3 / 2} \eta^{2}(\lambda)\right) .
\end{aligned}
$$

\section{Proof of the main results}


Asymptotics of Eigenvalues for Sturm-Liouville Problem with Eigenvalue in the Boundary Condition for Differentiable Potential

\section{Proof of Theorem 1.}

i) If $\mathrm{a}_{2}^{\prime} \neq 0$ and $\beta \neq 0$, the real solution of $\mathrm{y}^{\prime \prime}(\mathrm{t})+[\lambda-\mathrm{q}(\mathrm{t})] \mathrm{y}(\mathrm{t})=0$ is $y(t, \lambda)=R(t, \lambda) \cos \theta(t, \lambda)$ from (6). We use this equation for boundary $t=a$, we find $\mathrm{R}(\mathrm{a}, \lambda)\left\{\cos \theta(\mathrm{a}, \lambda)\left[\left(-\mathrm{a}_{2}^{\prime} \lambda+\mathrm{a}_{2}\right) \frac{\mathrm{R}^{\prime}(\mathrm{a}, \lambda)}{\mathrm{R}(\mathrm{a}, \lambda)}-\left(-\mathrm{a}_{1}^{\prime} \lambda+\mathrm{a}_{1}\right)\right]-\left(-\mathrm{a}_{2}^{\prime} \lambda+\mathrm{a}_{2}\right) \theta^{\prime}(\mathrm{a}, \lambda) \sin \theta(\mathrm{a}, \lambda)\right\}$ $=0$.

If we choose $\alpha_{1}$ as

$$
\begin{aligned}
& \sin \alpha_{1}:=\left(-a_{2}^{\prime} \lambda+a_{2}\right) \frac{R^{\prime}(a, \lambda)}{R(a, \lambda)}-\left(-a_{1}^{\prime} \lambda+a_{1}\right), \\
& \cos \alpha_{1}:=-\left(-a_{2}^{\prime} \lambda+a_{2}\right) \theta^{\prime}(a, \lambda),
\end{aligned}
$$

we have

$$
\mathrm{R}(\mathrm{a}, \lambda) \sin \left[\alpha_{1}+\theta(\mathrm{a}, \lambda)\right]=0 \operatorname{sosin}\left[\alpha_{1}+\theta(\mathrm{a}, \lambda)\right]=0 \text { or } \theta(\mathrm{a}, \lambda)=-\alpha_{1} .
$$

Using by equations (7) and (8) as

$$
\mathrm{S}(\mathrm{t}, \lambda)=\frac{\mathrm{R}^{\prime}(\mathrm{t}, \lambda)}{\mathrm{R}(\mathrm{t}, \lambda)}, \mathrm{T}(\mathrm{t}, \lambda)=\theta^{\prime}(\mathrm{t}, \lambda)
$$

and their asymptotic expansions (14)-(15), we calculate

$$
\begin{aligned}
a_{1}^{\prime} \lambda+\frac{1}{2} \lambda^{1 / 2} a_{2}^{\prime} q(b) \sin 2 \lambda^{1 / 2}(b-a)-\frac{1}{2} \lambda^{1 / 2} a_{2}^{\prime} \cos \left(2 \lambda^{1 / 2} a+\xi_{a}\right)-a_{1} \\
\frac{\sin \alpha_{1}}{\cos \alpha_{1}}=\frac{+\frac{1}{2} \lambda^{1 / 2} a_{2} q(b) \sin 2 \lambda^{1 / 2}(b-a)+\frac{1}{2} \lambda^{-1 / 2} a_{2} \cos \left(2 \lambda^{1 / 2} a+\xi_{a}\right)}{a_{2}^{\prime} \lambda^{3 / 2}+\frac{1}{2} \lambda^{1 / 2} a_{2}^{\prime} q(b) \cos 2 \lambda^{1 / 2}(b-a)-\frac{1}{2} \lambda^{1 / 2} a_{2}^{\prime} q(a)} \\
-\frac{1}{2} \lambda^{1 / 2} a_{2}^{\prime} \sin \left(2 \lambda^{1 / 2} a+\xi_{a}\right)-a_{2} \eta^{1 / 2}-\frac{1}{2} \lambda^{-1 / 2} a_{2} q(b) \cos 2 \lambda^{1 / 2}(b-a) \\
+\frac{1}{2} \lambda^{-1 / 2} a_{2} q(a)+\frac{1}{2} \lambda^{-1 / 2} a_{2} \sin \left(2 \lambda^{1 / 2} a+\xi_{a}\right)+O\left(\eta^{2}(\lambda)\right)+O\left(\lambda^{-1} \eta^{2}(\lambda)\right)
\end{aligned}
$$

so 


\section{Haskız Coşkun and Elif Başkaya}

$$
\begin{gathered}
\frac{a_{1}^{\prime} \lambda+\frac{1}{2} \lambda^{1 / 2} a_{2}^{\prime} q(b) \sin 2 \lambda^{1 / 2}(b-a)-\frac{1}{2} \lambda^{1 / 2} a_{2}^{\prime} \cos \left(2 \lambda^{1 / 2} a+\xi_{a}\right)-a_{1}}{\cos \alpha_{1}}=\frac{+O\left(\eta^{2}(\lambda)\right)+O\left(\lambda^{-1} \eta^{2}(\lambda)\right)}{-\frac{1}{2} \lambda^{1 / 2} a_{2} q(b) \sin 2 \lambda^{1 / 2}(b-a)+\frac{1}{2} \lambda^{-1 / 2} a_{2} \cos \left(2 \lambda^{1 / 2} a+\xi_{a}\right)} \\
{\left[\begin{array}{l}
1+\frac{1}{2} \lambda^{-1} q(b) \cos 2 \lambda^{1 / 2}(b-a)-\frac{1}{2} \lambda^{-1} q(a) \\
-\frac{1}{2} \lambda^{-1} \sin \left(2 \lambda^{1 / 2} a+\xi_{a}\right)-\frac{a_{2}}{a_{2}^{\prime}} \lambda^{-1}-\frac{1}{2} \lambda^{-2} \frac{a_{2}}{a_{2}^{\prime}} q(b) \cos 2 \lambda^{1 / 2}(b-a) \\
+\frac{1}{2} \lambda^{-2} \frac{a_{2}}{a_{2}^{\prime}} q(a)+\frac{1}{2} \lambda^{-2} \frac{a_{2}}{a_{2}^{\prime}} \sin \left(2 \lambda^{1 / 2} a+\xi_{a}\right)+O\left(\lambda^{-3 / 2} \eta^{2}(\lambda)\right)
\end{array}\right]}
\end{gathered}
$$

Then

$$
\begin{aligned}
\tan \alpha_{1} & =\left[\begin{array}{l}
\frac{a_{1}^{\prime}}{a_{2}^{\prime}} \lambda^{-1 / 2}+\frac{1}{2} \lambda^{-1} q(b) \sin 2 \lambda^{1 / 2}(b-a)-\frac{1}{2} \lambda^{-1} \cos \left(2 \lambda^{1 / 2} \mathrm{a}+\xi_{\mathrm{a}}\right)-\frac{\mathrm{a}_{1}}{\mathrm{a}_{2}^{\prime}} \lambda^{-3 / 2} \\
-\frac{\mathrm{a}_{2}}{2 \mathrm{a}_{2}^{\prime}} \lambda^{-2} \mathrm{q}(\mathrm{b}) \sin 2 \lambda^{1 / 2}(\mathrm{~b}-\mathrm{a})+\frac{\mathrm{a}_{2}}{2 \mathrm{a}_{2}^{\prime}} \lambda^{-2} \cos \left(2 \lambda^{1 / 2} \mathrm{a}+\xi_{\mathrm{a}}\right)+O\left(\lambda^{-3 / 2} \eta^{2}(\lambda)\right)
\end{array}\right] \\
\times & {\left[\begin{array}{l}
1-\frac{1}{2} \lambda^{-1} \mathrm{q}(\mathrm{b}) \cos 2 \lambda^{1 / 2}(\mathrm{~b}-\mathrm{a})+\frac{1}{2} \lambda^{-1} \mathrm{q}(\mathrm{a})+\frac{1}{2} \lambda^{-1} \sin \left(2 \lambda^{1 / 2} \mathrm{a}+\xi_{\mathrm{a}}\right) \\
+\frac{\mathrm{a}_{2}}{\mathrm{a}_{2}^{\prime}} \lambda^{-1}+\frac{1}{2} \lambda^{-2} \frac{\mathrm{a}_{2}}{\mathrm{a}_{2}^{\prime}} \mathrm{q}(\mathrm{b}) \cos 2 \lambda^{1 / 2}(\mathrm{~b}-\mathrm{a})-\frac{1}{2} \lambda^{-2} \frac{\mathrm{a}_{2}}{\mathrm{a}_{2}^{\prime}} \mathrm{q}(\mathrm{a}) \\
-\frac{1}{2} \lambda^{-2} \frac{\mathrm{a}_{2}}{\mathrm{a}_{2}^{\prime}} \sin \left(2 \lambda^{1 / 2} \mathrm{a}+\xi_{\mathrm{a}}\right)+\frac{1}{4} \lambda^{-2} \mathrm{q}^{2}(\mathrm{a})+\frac{1}{4} \lambda^{-2} \sin ^{2}\left(2 \lambda^{1 / 2} \mathrm{a}+\xi_{\mathrm{a}}\right) \\
+\left[\frac{\mathrm{a}_{2}}{\mathrm{a}_{2}^{\prime}}\right]^{2} \lambda^{-2}-\frac{1}{2} \lambda^{-2} \mathrm{q}(\mathrm{a}) \mathrm{q}(\mathrm{b}) \cos 2 \lambda^{1 / 2}(\mathrm{~b}-\mathrm{a})+\frac{\mathrm{a}_{2}}{\mathrm{a}_{2}^{\prime}} \lambda^{-2} \mathrm{q}(\mathrm{a}) \\
-\frac{1}{2} \lambda^{-2} \mathrm{q}(\mathrm{b}) \cos 2 \lambda^{1 / 2}(\mathrm{~b}-\mathrm{a}) \sin \left(2 \lambda^{1 / 2} \mathrm{a}+\xi_{\mathrm{a}}\right)-\frac{\mathrm{a}_{2}}{\mathrm{a}_{2}^{\prime}} \lambda^{-2} \mathrm{q}(\mathrm{b}) \cos 2 \lambda^{1 / 2}(\mathrm{~b}-\mathrm{a}) \\
+\frac{1}{2} \lambda^{-2} \mathrm{q}(\mathrm{a}) \sin \left(2 \lambda^{1 / 2} \mathrm{a}+\xi_{\mathrm{a}}\right)+\frac{\mathrm{a}_{2}}{\mathrm{a}_{2}^{\prime}} \lambda^{-2} \sin \left(2 \lambda^{1 / 2} \mathrm{a}+\xi_{\mathrm{a}}\right)+O\left(\lambda^{-3 / 2} \eta^{2}(\lambda)\right)
\end{array}\right], }
\end{aligned}
$$

hence 
Asymptotics of Eigenvalues for Sturm-Liouville Problem with Eigenvalue in the Boundary Condition for Differentiable Potential

$$
\begin{aligned}
\tan \alpha_{1}= & \frac{a_{1}^{\prime}}{a_{2}^{\prime}} \lambda^{-1 / 2}+\frac{1}{2} \lambda^{-1} q(b) \sin 2 \lambda^{1 / 2}(b-a)-\frac{1}{2} \lambda^{-1} \cos \left(2 \lambda^{1 / 2} \mathrm{a}+\xi_{\mathrm{a}}\right)-\frac{\mathrm{a}_{1}}{\mathrm{a}_{2}^{\prime}} \lambda^{-3 / 2} \\
& -\frac{\mathrm{a}_{2}}{2 \mathrm{a}_{2}^{\prime}} \lambda^{-2} \mathrm{q}(\mathrm{b}) \sin 2 \lambda^{1 / 2}(\mathrm{~b}-\mathrm{a})+\frac{\mathrm{a}_{2}}{2 \mathrm{a}_{2}^{\prime}} \lambda^{-2} \cos \left(2 \lambda^{1 / 2} \mathrm{a}+\xi_{\mathrm{a}}\right) \\
& +\frac{\mathrm{a}_{1}^{\prime}}{2 \mathrm{a}_{2}^{\prime}} \lambda^{-3 / 2} \mathrm{q}(\mathrm{b}) \cos 2 \lambda^{1 / 2}(\mathrm{~b}-\mathrm{a})+\frac{\mathrm{a}_{1}^{\prime}}{2 \mathrm{a}_{2}^{\prime}} \lambda^{-3 / 2} \mathrm{q}(\mathrm{a})+\frac{\mathrm{a}_{1}^{\prime}}{2 \mathrm{a}_{2}^{\prime}} \lambda^{-3 / 2} \sin \left(2 \lambda^{1 / 2} \mathrm{a}+\xi_{\mathrm{a}}\right) \\
& +\frac{\mathrm{a}_{1}^{\prime} \mathrm{a}_{2}}{\left[\mathrm{a}_{2}^{\prime}\right]^{2}} \lambda^{-3 / 2}-\frac{1}{4} \lambda^{-2} \mathrm{q}^{2}(\mathrm{~b}) \cos 2 \lambda^{1 / 2}(\mathrm{~b}-\mathrm{a}) \sin 2 \lambda^{1 / 2}(\mathrm{~b}-\mathrm{a}) \\
& +\frac{1}{4} \lambda^{-2} \mathrm{q}(\mathrm{a}) \mathrm{q}(\mathrm{b}) \sin 2 \lambda^{1 / 2}(\mathrm{~b}-\mathrm{a})+\frac{1}{4} \lambda^{-2} \mathrm{q}(\mathrm{b}) \sin 2 \lambda^{1 / 2}(\mathrm{~b}-\mathrm{a}) \sin \left(2 \lambda^{1 / 2} \mathrm{a}+\xi_{\mathrm{a}}\right) \\
& +\frac{\mathrm{a}_{2}}{2 \mathrm{a}_{2}^{\prime}} \lambda^{-2} \mathrm{q}(\mathrm{b}) \sin 2 \lambda^{1 / 2}(\mathrm{~b}-\mathrm{a})+\frac{1}{4} \lambda^{-2} \mathrm{q}(\mathrm{b}) \cos 2 \lambda^{1 / 2}(\mathrm{~b}-\mathrm{a}) \cos \left(2 \lambda^{1 / 2} \mathrm{a}+\xi_{\mathrm{a}}\right) \\
& -\frac{\mathrm{a}_{2}}{2 \mathrm{a}_{2}^{\prime}} \lambda^{-2} \cos \left(2 \lambda^{1 / 2} \mathrm{a}+\xi_{\mathrm{a}}\right)-\frac{1}{4} \lambda^{-2} \mathrm{q}(\mathrm{a}) \cos \left(2 \lambda^{1 / 2} \mathrm{a}+\xi_{\mathrm{a}}\right)+O\left(\lambda^{-3 / 2} \eta^{2}(\lambda)\right) .
\end{aligned}
$$

In the last equation, by using Taylor expansion of $\arctan \mathrm{x}$ at $\mathrm{x}=0$, we obtain

$$
\begin{aligned}
\alpha_{1} & =\frac{\mathrm{a}_{1}^{\prime}}{\mathrm{a}_{2}^{\prime}} \lambda^{-1 / 2}+\frac{1}{2} \lambda^{-1} \mathrm{q}(\mathrm{b}) \sin 2 \lambda^{1 / 2}(\mathrm{~b}-\mathrm{a})-\frac{1}{2} \lambda^{-1} \cos \left(2 \lambda^{1 / 2} \mathrm{a}+\xi_{\mathrm{a}}\right)-\frac{\mathrm{a}_{1}}{\mathrm{a}_{2}^{\prime}} \lambda^{-3 / 2} \\
& -\frac{1}{3}\left[\frac{\mathrm{a}_{1}^{\prime}}{\mathrm{a}_{2}^{\prime}}\right]^{3} \lambda^{-3 / 2}-\frac{1}{2}\left[\frac{\mathrm{a}_{1}^{\prime}}{\mathrm{a}_{2}^{\prime}}\right]^{2} \lambda^{-3 / 2} \mathrm{q}(\mathrm{b}) \sin 2 \lambda^{1 / 2}(\mathrm{~b}-\mathrm{a}) \\
& +\frac{\mathrm{a}_{1}^{\prime}}{2 \mathrm{a}_{2}^{\prime}} \lambda^{-3 / 2} \mathrm{q}(\mathrm{b}) \cos 2 \lambda^{1 / 2}(\mathrm{~b}-\mathrm{a})+\frac{\mathrm{a}_{1}^{\prime}}{2 \mathrm{a}_{2}^{\prime}} \lambda^{-3 / 2} \mathrm{q}(\mathrm{a}) \\
& +\frac{\mathrm{a}_{1}^{\prime}}{2 \mathrm{a}_{2}^{\prime}} \lambda^{-3 / 2} \sin \left(2 \lambda^{1 / 2} \mathrm{a}+\xi_{\mathrm{a}}\right)+\frac{\mathrm{a}_{1}^{\prime} \mathrm{a}_{2}}{\left[\mathrm{a}_{2}^{\prime}\right]^{2}} \lambda^{-3 / 2}+\frac{1}{4} \lambda^{-2} \mathrm{q}(\mathrm{a}) \mathrm{q}(\mathrm{b}) \sin 2 \lambda^{1 / 2}(\mathrm{~b}-\mathrm{a}) \\
& -\frac{1}{4} \lambda^{-2} \mathrm{q}^{2}(\mathrm{~b}) \cos 2 \lambda^{1 / 2}(\mathrm{~b}-\mathrm{a}) \sin 2 \lambda^{1 / 2}(\mathrm{~b}-\mathrm{a})-\frac{\mathrm{a}_{2}}{2 \mathrm{a}_{2}^{\prime}} \lambda^{-2} \cos \left(2 \lambda^{1 / 2} \mathrm{a}+\xi_{\mathrm{a}}\right) \\
& +\frac{1}{4} \lambda^{-2} \mathrm{q}(\mathrm{b}) \sin 2 \lambda^{1 / 2}(\mathrm{~b}-\mathrm{a}) \sin \left(2 \lambda^{1 / 2} \mathrm{a}+\xi_{\mathrm{a}}\right) \\
& +\frac{1}{4} \lambda^{-2} \mathrm{q}(\mathrm{b}) \cos 2 \lambda^{1 / 2}(\mathrm{~b}-\mathrm{a}) \cos \left(2 \lambda^{1 / 2} \mathrm{a}+\xi_{\mathrm{a}}\right) \\
& -\frac{1}{4} \lambda^{-2} \mathrm{q}(\mathrm{a}) \cos \left(2 \lambda^{1 / 2} \mathrm{a}+\xi_{\mathrm{a}}\right)+\mathrm{O}\left(\lambda^{-3 / 2} \eta^{2}(\lambda)\right) .
\end{aligned}
$$

When we use the form $y(t, \lambda)=R(t, \lambda) \cos \theta(t, \lambda)$ for boundary $t=b$, we find

If we choose $\alpha_{2}$ as

$$
\mathrm{R}(\mathrm{b}, \lambda)\left\{\cos \theta(\mathrm{b}, \lambda)\left[\cos \beta+\frac{\mathrm{R}^{\prime}(\mathrm{b}, \lambda)}{\mathrm{R}(\mathrm{b}, \lambda)} \sin \beta\right]-\sin \theta(\mathrm{b}, \lambda) \theta^{\prime}(\mathrm{b}, \lambda) \sin \beta\right\}=0 .
$$




\section{Haskız Coşkun and Elif Başkaya}

$$
\begin{gathered}
\sin \alpha_{2}:=\cos \beta+\frac{\mathrm{R}^{\prime}(\mathrm{b}, \lambda)}{\mathrm{R}(\mathrm{b}, \lambda)} \sin \beta, \\
\cos \alpha_{2}:=\theta^{\prime}(\mathrm{b}, \lambda) \sin \beta,
\end{gathered}
$$

we have $R(b, \lambda) \sin \left[\alpha_{2}-\theta(b, \lambda)\right]=0$ so $\sin \left[\alpha_{2}-\theta(b, \lambda)\right]=0$ or $\theta(b, \lambda)=\alpha_{2}+(n+1) \pi$.

Using by $S(t, \lambda)=\frac{R^{\prime}(t, \lambda)}{R(t, \lambda)}, T(t, \lambda)=\theta^{\prime}(t, \lambda)$ and their asymptotic expansions (14)-(15), we can write

$$
\frac{\sin \alpha_{2}}{\cos \alpha_{2}}=\frac{\cos \beta+O\left(\lambda^{-1} \eta^{2}(\lambda)\right)}{\lambda^{1 / 2} \sin \beta+O\left(\lambda^{-1} \eta^{2}(\lambda)\right)}=\frac{\cos \beta+O\left(\lambda^{-1} \eta^{2}(\lambda)\right)}{\lambda^{1 / 2} \sin \beta\left[1+O\left(\lambda^{-3 / 2} \eta^{2}(\lambda)\right)\right]},
$$

so

$$
\begin{aligned}
\tan \alpha_{2} & =\left[\lambda^{1 / 2} \cot \beta+O\left(\lambda^{-3 / 2} \eta^{2}(\lambda)\right)\right]\left[1+O\left(\lambda^{-3 / 2} \eta^{2}(\lambda)\right)\right] \\
& =\lambda^{1 / 2} \cot \beta+O\left(\lambda^{-3 / 2} \eta^{2}(\lambda)\right) .
\end{aligned}
$$

In the last equation, by using Taylor expansion of $\arctan \mathrm{x}$ at $\mathrm{x}=0$, we obtain

$$
\alpha_{2}=\lambda^{1 / 2} \cot \beta-\frac{1}{3} \lambda^{-3 / 2} \cot ^{3} \beta+O\left(\lambda^{-3 / 2} \eta^{2}(\lambda)\right) \text {. }
$$

Let use these findings (16), (17) and (20) in (13), we see that

$$
\begin{aligned}
& \frac{(2 \mathrm{n}+3) \pi}{2}+\frac{\mathrm{a}_{1}^{\prime}}{\mathrm{a}_{2}^{\prime}} \lambda^{-1 / 2}+\frac{1}{2} \lambda^{-1} \mathrm{q}(\mathrm{b}) \sin 2 \lambda^{1 / 2}(\mathrm{~b}-\mathrm{a})-\frac{1}{2} \lambda^{-1} \cos \left(2 \lambda^{1 / 2} \mathrm{a}+\xi_{\mathrm{a}}\right) \\
& -\frac{\mathrm{a}_{1}}{\mathrm{a}_{2}^{\prime}} \lambda^{-3 / 2}-\frac{1}{3}\left[\frac{\mathrm{a}_{1}^{\prime}}{\mathrm{a}_{2}^{\prime}}\right]^{3} \lambda^{-3 / 2}-\frac{1}{2}\left[\frac{\mathrm{a}_{1}^{\prime}}{\mathrm{a}_{2}^{\prime}}\right]^{2} \lambda^{-3 / 2} \mathrm{q}(\mathrm{b}) \sin 2 \lambda^{1 / 2}(\mathrm{~b}-\mathrm{a}) \\
& +\frac{\mathrm{a}_{1}^{\prime}}{2 \mathrm{a}_{2}^{\prime}} \lambda^{-3 / 2} \mathrm{q}(\mathrm{b}) \cos 2 \lambda^{1 / 2}(\mathrm{~b}-\mathrm{a})+\frac{\mathrm{a}_{1}^{\prime}}{2 \mathrm{a}_{2}^{\prime}} \lambda^{-3 / 2} \mathrm{q}(\mathrm{a}) \\
& +\frac{\mathrm{a}_{1}^{\prime}}{2 \mathrm{a}_{2}^{\prime}} \lambda^{-3 / 2} \sin \left(2 \lambda^{1 / 2} \mathrm{a}+\xi_{\mathrm{a}}\right)+\frac{\mathrm{a}_{1}^{\prime} \mathrm{a}_{2}}{\left[\mathrm{a}_{2}^{\prime}\right]^{2}} \lambda^{-3 / 2}+\frac{1}{4} \lambda^{-2} \mathrm{q}(\mathrm{a}) \mathrm{q}(\mathrm{b}) \sin 2 \lambda^{1 / 2}(\mathrm{~b}-\mathrm{a}) \\
& -\frac{1}{4} \lambda^{-2} \mathrm{q}^{2}(\mathrm{~b}) \cos 2 \lambda^{1 / 2}(\mathrm{~b}-\mathrm{a}) \sin 2 \lambda^{1 / 2}(\mathrm{~b}-\mathrm{a})+O\left(\lambda^{-3 / 2} \eta^{2}(\lambda)\right) \\
& =\lambda^{1 / 2}(\mathrm{~b}-\mathrm{a})+\frac{1}{4} \lambda^{-1} \mathrm{q}(\mathrm{b}) \sin 2 \lambda^{1 / 2}(\mathrm{~b}-\mathrm{a})-\frac{1}{4} \lambda^{-1} \cos \left(2 \lambda^{1 / 2} \mathrm{a}+\xi_{\mathrm{a}}\right)+O\left(\lambda^{-2} \eta(\lambda)\right) .
\end{aligned}
$$

We prove the theorem by using definitions of $\sin \xi_{\mathrm{t}}, \cos \xi_{\mathrm{t}}$ and $\eta(\lambda)$; also series error computation in the last equation.

\section{Proof of Theorem 2.}

ii) If $\mathrm{a}_{2}^{\prime}=0$ and $\beta=0$, the real solution of $\mathrm{y}^{\prime \prime}(\mathrm{t})+[\lambda-\mathrm{q}(\mathrm{t})] \mathrm{y}(\mathrm{t})=0$ is $\mathrm{y}(\mathrm{t}, \lambda)=\mathrm{R}(\mathrm{t}, \lambda) \cos \theta(\mathrm{t}, \lambda)$. We use this equation for boundary $\mathrm{t}=\mathrm{a}$, we find 
Asymptotics of Eigenvalues for Sturm-Liouville Problem with Eigenvalue in the Boundary Condition for Differentiable Potential

$\mathrm{R}(\mathrm{a}, \lambda)\left\{\cos \theta(\mathrm{a}, \lambda)\left[\frac{\mathrm{R}^{\prime}(\mathrm{a}, \lambda)}{\mathrm{R}(\mathrm{a}, \lambda)}+\frac{\mathrm{a}_{1}^{\prime} \lambda-\mathrm{a}_{1}}{\mathrm{a}_{2}}\right]-\theta^{\prime}(\mathrm{a}, \lambda) \sin \theta(\mathrm{a}, \lambda)\right\}=0$.

If we choose $\alpha_{3}$ as

$$
\begin{aligned}
& \sin \alpha_{3}:=\frac{\mathrm{R}^{\prime}(\mathrm{a}, \lambda)}{\mathrm{R}(\mathrm{a}, \lambda)}+\frac{\mathrm{a}_{1}^{\prime} \lambda-\mathrm{a}_{1}}{\mathrm{a}_{2}}, \\
& \cos \alpha_{3}:=-\theta^{\prime}(\mathrm{a}, \lambda),
\end{aligned}
$$

we have $\mathrm{R}(\mathrm{a}, \lambda) \sin \left[\alpha_{3}+\theta(\mathrm{a}, \lambda)\right]=0$ so $\sin \left[\alpha_{3}+\theta(\mathrm{a}, \lambda)\right]=0$ or $\theta(\mathrm{a}, \lambda)=-\alpha_{3}$. Using by

$$
\mathrm{S}(\mathrm{t}, \lambda)=\frac{\mathrm{R}^{\prime}(\mathrm{t}, \lambda)}{\mathrm{R}(\mathrm{t}, \lambda)}, \mathrm{T}(\mathrm{t}, \lambda)=\theta^{\prime}(\mathrm{t}, \lambda)
$$

and their asymptotic expansions (14)-(15), one writes

$$
\begin{aligned}
\cot \alpha_{3}=\left[\begin{array}{l}
-\frac{\mathrm{a}_{2}}{\mathrm{a}_{1}^{\prime}} \lambda^{-1 / 2}-\frac{\mathrm{a}_{2}}{2 \mathrm{a}_{1}^{\prime}} \lambda^{-3 / 2} \mathrm{q}(\mathrm{b}) \cos 2 \lambda^{1 / 2}(\mathrm{~b}-\mathrm{a})+\frac{\mathrm{a}_{2}}{2 \mathrm{a}_{1}^{\prime}} \lambda^{-3 / 2} \sin \left(2 \lambda^{1 / 2} \mathrm{a}+\xi_{\mathrm{a}}\right) \\
+\frac{\mathrm{a}_{2}}{2 \mathrm{a}_{1}^{\prime}} \lambda^{-3 / 2} \mathrm{q}(\mathrm{a})+\mathrm{O}\left(\lambda^{-2} \eta^{2}(\lambda)\right)
\end{array}\right] \\
\times\left[\begin{array}{l}
1+\frac{\mathrm{a}_{1}}{\mathrm{a}_{1}^{\prime}} \lambda^{-1}+\frac{\mathrm{a}_{2}}{2 \mathrm{a}_{1}^{\prime}} \lambda^{-3 / 2} \mathrm{q}(\mathrm{b}) \sin 2 \lambda^{1 / 2}(\mathrm{~b}-\mathrm{a})-\frac{\mathrm{a}_{2}}{2 \mathrm{a}_{1}^{\prime}} \lambda^{-3 / 2} \cos \left(2 \lambda^{1 / 2} \mathrm{a}+\xi_{\mathrm{a}}\right) \\
+\left[\frac{\mathrm{a}_{1}}{\mathrm{a}_{1}^{\prime}}\right]^{2} \lambda^{-2}-\frac{\mathrm{a}_{1} \mathrm{a}_{2}}{\left[\mathrm{a}_{1}^{\prime}\right]^{2}} \lambda^{-5 / 2} \mathrm{q}(\mathrm{b}) \sin 2 \lambda^{1 / 2}(\mathrm{~b}-\mathrm{a}) \\
+\frac{\mathrm{a}_{1} \mathrm{a}_{2}}{\left[\mathrm{a}_{1}^{\prime}\right]^{2}} \lambda^{-5 / 2} \cos \left(2 \lambda^{1 / 2} \mathrm{a}+\xi_{\mathrm{a}}\right)+\mathrm{O}\left(\lambda^{-2} \eta^{2}(\lambda)\right)
\end{array}\right],
\end{aligned}
$$

Then

$$
\begin{aligned}
\cot \alpha_{3} & =-\frac{\mathrm{a}_{2}}{\mathrm{a}_{1}^{\prime}} \lambda^{-1 / 2}-\frac{\mathrm{a}_{2}}{2 \mathrm{a}_{1}^{\prime}} \lambda^{-3 / 2} \mathrm{q}(\mathrm{b}) \cos 2 \lambda^{1 / 2}(\mathrm{~b}-\mathrm{a})+\frac{\mathrm{a}_{2}}{2 \mathrm{a}_{1}^{\prime}} \lambda^{-3 / 2} \sin \left(2 \lambda^{1 / 2} \mathrm{a}+\xi_{\mathrm{a}}\right) \\
& +\frac{\mathrm{a}_{2}}{2 \mathrm{a}_{1}^{\prime}} \lambda^{-3 / 2} \mathrm{q}(\mathrm{a})-\frac{\mathrm{a}_{1} \mathrm{a}_{2}}{\left[\mathrm{a}_{1}^{\prime}\right]^{2}} \lambda^{-3 / 2}-\frac{\mathrm{a}_{2}^{2}}{2\left[\mathrm{a}_{1}^{\prime}\right]^{2}} \lambda^{-2} \mathrm{q}(\mathrm{b}) \sin 2 \lambda^{1 / 2}(\mathrm{~b}-\mathrm{a})-\frac{\mathrm{a}_{1}^{2} \mathrm{a}_{2}}{\left[\mathrm{a}_{1}^{\prime}\right]^{3}} \lambda^{-5 / 2} \\
& +\frac{\mathrm{a}_{2}^{2}}{2\left[\mathrm{a}_{1}^{\prime}\right]^{2}} \lambda^{-2} \cos \left(2 \lambda^{1 / 2} \mathrm{a}+\xi_{\mathrm{a}}\right)-\frac{\mathrm{a}_{1} \mathrm{a}_{2}}{2\left[\mathrm{a}_{1}^{\prime}\right]^{2}} \lambda^{-5 / 2} \mathrm{q}(\mathrm{b}) \cos 2 \lambda^{1 / 2}(\mathrm{~b}-\mathrm{a}) \\
& +\frac{\mathrm{a}_{1} \mathrm{a}_{2}}{2\left[\mathrm{a}_{1}^{\prime}\right]^{2}} \lambda^{-5 / 2} \mathrm{q}(\mathrm{a})+\frac{\mathrm{a}_{1} \mathrm{a}_{2}}{2\left[\mathrm{a}_{1}^{\prime}\right]^{2}} \lambda^{-5 / 2} \sin \left(2 \lambda^{1 / 2} \mathrm{a}+\xi_{\mathrm{a}}\right)+\mathrm{O}\left(\lambda^{-2} \eta^{2}(\lambda)\right) .
\end{aligned}
$$

In the last equation, by using Taylor expansion $\operatorname{arccot} \mathrm{x}$ at $\mathrm{x}=0$, we obtain 
Haskız Coşkun and Elif Başkaya

$$
\begin{gathered}
-\theta(\mathrm{a}, \lambda)=\alpha_{3}=\frac{\pi}{2}+\frac{\mathrm{a}_{2}}{\mathrm{a}_{1}^{\prime}} \lambda^{-1 / 2}+\frac{\mathrm{a}_{2}}{2 \mathrm{a}_{1}^{\prime}} \lambda^{-3 / 2} \mathrm{q}(\mathrm{b}) \cos 2 \lambda^{1 / 2}(\mathrm{~b}-\mathrm{a})-\frac{\mathrm{a}_{2}}{2 \mathrm{a}_{1}^{\prime}} \lambda^{-3 / 2} \sin \left(2 \lambda^{1 / 2} \mathrm{a}+\xi_{\mathrm{a}}\right) \\
-\frac{\mathrm{a}_{2}}{2 \mathrm{a}_{1}^{\prime}} \lambda^{-3 / 2} \mathrm{q}(\mathrm{a})+\frac{\mathrm{a}_{1} \mathrm{a}_{2}}{\left[\mathrm{a}_{1}^{\prime}\right]^{2}} \lambda^{-3 / 2}+\frac{\mathrm{a}_{2}^{2}}{2\left[\mathrm{a}_{1}^{\prime}\right]^{2}} \lambda^{-2} \mathrm{q}(\mathrm{b}) \sin 2 \lambda^{1 / 2}(\mathrm{~b}-\mathrm{a})+\frac{\mathrm{a}_{1}^{2} \mathrm{a}_{2}}{\left[\mathrm{a}_{1}^{\prime}\right]^{3}} \lambda^{-5 / 2} \\
-\frac{\mathrm{a}_{2}^{2}}{2\left[\mathrm{a}_{1}^{\prime}\right]^{2}} \lambda^{-2} \cos \left(2 \lambda^{1 / 2} \mathrm{a}+\xi_{\mathrm{a}}\right)+\frac{\mathrm{a}_{1} \mathrm{a}_{2}}{2\left[\mathrm{a}_{1}^{\prime}\right]^{2}} \lambda^{-5 / 2} \mathrm{q}(\mathrm{b}) \cos 2 \lambda^{1 / 2}(\mathrm{~b}-\mathrm{a}) \\
-\frac{\mathrm{a}_{1} \mathrm{a}_{2}}{2\left[\mathrm{a}_{1}^{\prime}\right]^{2}} \lambda^{-5 / 2} \mathrm{q}(\mathrm{a})-\frac{\mathrm{a}_{1} \mathrm{a}_{2}}{2\left[\mathrm{a}_{1}^{\prime}\right]^{2}} \lambda^{-5 / 2} \sin \left(2 \lambda^{1 / 2} \mathrm{a}+\xi_{\mathrm{a}}\right)-\frac{\mathrm{a}_{2}^{3}}{3\left[\mathrm{a}_{1}^{\prime}\right]^{3}} \lambda^{-3 / 2}+O\left(\lambda^{-2} \eta^{2}(\lambda)\right)
\end{gathered}
$$

For boundary $\mathrm{t}=\mathrm{b}$, by using $\mathrm{y}(\mathrm{t}, \lambda)=\mathrm{R}(\mathrm{t}, \lambda) \cos \theta(\mathrm{t}, \lambda)$ and $\beta=0$, we have $\cos \theta(\mathrm{t}, \lambda)=0$, so

$$
\theta(\mathrm{b}, \lambda)=\frac{\pi}{2}+(\mathrm{n}+1) \pi
$$

Let use these findings in $\theta(b, \lambda)-\theta(a, \lambda)=\lambda^{1 / 2}(b-a)+\sum_{n=1}^{\infty} \operatorname{Im} \int_{a}^{b} v_{n}(x, \lambda) d x$, we estimate that

$$
\begin{aligned}
& (\mathrm{n}+2) \pi+\frac{\mathrm{a}_{2}}{\mathrm{a}_{1}^{\prime}} \lambda^{-1 / 2}+\frac{\mathrm{a}_{2}}{2 \mathrm{a}_{1}^{\prime}} \lambda^{-3 / 2} \mathrm{q}(\mathrm{b}) \cos 2 \lambda^{1 / 2}(\mathrm{~b}-\mathrm{a})-\frac{\mathrm{a}_{2}}{2 \mathrm{a}_{1}^{\prime}} \lambda^{-3 / 2} \sin \left(2 \lambda^{1 / 2} \mathrm{a}+\xi_{\mathrm{a}}\right) \\
& -\frac{\mathrm{a}_{2}}{2 \mathrm{a}_{1}^{\prime}} \lambda^{-3 / 2} \mathrm{q}(\mathrm{a})+\frac{\mathrm{a}_{1} \mathrm{a}_{2}}{\left[\mathrm{a}_{1}^{\prime}\right]^{2}} \lambda^{-3 / 2}+\frac{\mathrm{a}_{2}^{2}}{2\left[\mathrm{a}_{1}^{\prime}\right]^{2}} \lambda^{-2} \mathrm{q}(\mathrm{b}) \sin 2 \lambda^{1 / 2}(\mathrm{~b}-\mathrm{a})+\frac{\mathrm{a}_{1}^{2} \mathrm{a}_{2}}{\left[\mathrm{a}_{1}^{\prime}\right]^{3}} \lambda^{-5 / 2} \\
& -\frac{\mathrm{a}_{2}^{2}}{2\left[\mathrm{a}_{1}^{\prime}\right]^{2}} \lambda^{-2} \cos \left(2 \lambda^{1 / 2} \mathrm{a}+\xi_{\mathrm{a}}\right)+\frac{\mathrm{a}_{1} \mathrm{a}_{2}}{2\left[\mathrm{a}_{1}^{\prime}\right]^{2}} \lambda^{-5 / 2} \mathrm{q}(\mathrm{b}) \cos 2 \lambda^{1 / 2}(\mathrm{~b}-\mathrm{a}) \\
& -\frac{\mathrm{a}_{1} \mathrm{a}_{2}}{2\left[\mathrm{a}_{1}^{\prime}\right]^{2}} \lambda^{-5 / 2} \mathrm{q}(\mathrm{a})-\frac{\mathrm{a}_{1} \mathrm{a}_{2}}{2\left[\mathrm{a}_{1}^{\prime}\right]^{2}} \lambda^{-5 / 2} \sin \left(2 \lambda^{1 / 2} \mathrm{a}+\xi_{\mathrm{a}}\right)-\frac{\mathrm{a}_{2}^{3}}{3\left[\mathrm{a}_{1}^{\prime}\right]^{3}} \lambda^{-3 / 2}+\mathrm{O}\left(\lambda^{-2} \eta^{2}(\lambda)\right) \\
& =\lambda^{1 / 2}(\mathrm{~b}-\mathrm{a})+\frac{1}{4} \lambda^{-1} \mathrm{q}(\mathrm{b}) \sin 2 \lambda^{1 / 2}(\mathrm{~b}-\mathrm{a})-\frac{1}{4} \lambda^{-1} \cos \left(2 \lambda^{1 / 2} \mathrm{a}+\xi_{\mathrm{a}}\right) \\
& +\mathrm{O}\left(\lambda^{-3 / 2} \eta^{2}(\lambda)\right) .
\end{aligned}
$$

We prove the theorem by using definitions of $\sin \xi_{t}, \cos \xi_{\mathrm{t}}$ and $\eta(\lambda)$; also series error computation in the last equation.

Similarly, Theorem 1-ii) follows from (13), (17) and (22); Theorem 2-i) follows from (13), (20) and (21).

\section{Conclusions}

In this paper, approximate eigenvalues are calculated for regular Sturm-Liouville problems having the eigenvalue parameter in the boundary condition with the potential that is continuous, also its differentiation exists and is integrable. 
Asymptotics of Eigenvalues for Sturm-Liouville Problem with Eigenvalue in the Boundary Condition for Differentiable Potential

\section{REFERENCES}

1. E.Başkaya, Regular Sturm-Liouville problems with eigenvalue parameter in the boundary conditions, $\mathrm{PhD}$. Thesis, Karadeniz Technical University, The Graduate School of Natural and Applied Sciences Biology Graduate Program, Trabzon, 2013.

2. H.Coşkun and N.Bayram, Asymptotics of eigenvalues for regular Sturm-Liouville problems with eigenvalue parameter in the boundary condition,Journal of Mathematical Analysis and Applications, 306 (2005) 548-566.

3. H.Coşkun and B.J.Harris, Estimates for the periodic and semi-periodic eigenvalues of Hill's equation, Proceedings of the royal society of Edinburgh section A: mathematics, 130 (2000) 991-998.

4. H.Coşkun, On the spectrum of a second-order periodic differential equation, Rocky Mountain Journal of Mathematics, 33 (2003) 1261-1277.

5. M.S.Eastham, The spectral theory of periodic differential equations, Scottish Academic Press, Edinburgh, 1973.

6. G.Fix, Asymptotic eigenvalues of Sturm-Liouville systems, Journal of Mathematical Analysis and Applications, 19 (1967) 519-525.

7. C.T.Fulton, An integral equation iterative scheme for asymptotic expansions of spectral quantities of regular Sturm-Liouville problems, Journal of Integral Equations, 4 (1982) 163-172.

8. C.T.Fulton, Two point boundary value problems with eigenvalue parameter contained in the boundary conditions, Proceedings of the Royal Society of Edinburgh Section A: Mathematics,77 (1977) 293-308.

9. C.T.Fulton and S.A.Pruess, Eigenvalue and eigen function asymptotics for regular Sturm-Liouville problems, Journal of Mathematical Analysis and Applications, 182 (1994) 297-340.

10. B.J.Harris, A series solution for certain Riccati equations with applications to SturmLiouville problems, Journal of Mathematical Analysis and Applications, 137 (1989) 462-470.

11. B.J.Harris, Asymptotics of eigenvalues for regular Sturm-Liouville problems, Journal of Mathematical Analysis and Applications, 183 (1994) 25-36.

12. B.J.Harris, The form of the spectral functions associated with Sturm-Liouville problems with continuous spectrum, Mathematika, 44 (1997) 149-161.

13. H.Hochstadt, Asymptotic estimates for the Sturm-Liouville spectrum, Communi cations on Pure and Applied Mathematics, 14 (1961) 749-764.

14. E.C.Titchmarsh, Eigenfunction expansions associated with second order differential equations I, $2^{\text {nd }}$ edn, Oxford University Press, Oxford, 1962.

15. J.Walter, Regular eigenvalue problems with eigenvalue parameter in the boundary condition, Mathematische Zeitschrift, 153 (1973) 301-312. 